\title{
Desarrollo de habilidades espaciales en estudiantes de ingeniería mediante CAD especializado
}

\author{
Development of spatial skills in engineering students by specialized CAD
}

\author{
J. A. Tristancho-Ortiz ID, L. F. Vargas-Tamayo iD, L. E. Contreras-Bravo iD
}

\begin{abstract}
Resumen-La habilidad espacial se puede definir como un componente de la inteligencia, ligada a la capacidad de formar una representación geométrica mental del mundo. Durante la escolarización el desarrollo de esta habilidad se integra con el currículo de la enseñanza matemática, pero generalmente la matemática se centra en el proceso algorítmico de solución de problemas matemáticos no espaciales, tanto en la primera infancia, educación formal o en la universidad. El dibujo de ingeniería se basa en la capacidad de manipular objetos e ideas mentalmente (usando las habilidades espaciales), para llevar a cabo el proceso de diseño de cualquier producto o proceso.

El presente artículo en la primera parte se hace una revisión de bibliográfica y del estado del arte. La segunda parte se muestra los resultados obtenidos en la aplicación de diferentes técnicas para el mejoramiento de las habilidades espaciales, identificando los puntos favorables y falencias, los cuales son usados como requisitos de necesidades para el desarrollo una nueva herramienta de software. En la parte final se establece el algoritmo diseñado, la implementación y se mide el impacto en un grupo de prueba.

Los resultados obtenidos muestran como esta nueva herramienta informática permite que se incremente de manera significativa la curva de aprendizaje y de desarrollo de habilidades espaciales.
\end{abstract}

Palabras claves - Dibujo asistido por computador (CAD), Habilidades espaciales, Pedagogía en dibujo de ingeniería, Tecnologías de la información y la comunicación (TIC).

Abstract - The Spatial ability can be defined as a component of intelligence, linked to the ability to form a mental geometric

Este manuscrito fue enviado el 30 de noviembre de 2018 y aceptado el 26 de marzo de 2019. Es un artículo de investigación científica derivado del proyecto de investigación "Generación y validación de contenidos didácticos para el desarrollo de habilidades espaciales de estudiantes de ingeniería por medio de la implementación de TIC", financiado por "Universidad Distrital Francisco José de Caldas" a través del "Instituto de Estudios e Investigaciones Educativas (IEIE)".

J. A. Tristancho-Ortiz. Profesor Asistente del proyecto curricular de Ingeniería Industrial de la Universidad Distrital Francisco José de Caldas. Docente investigador del grupo de investigación DIMSI (e-mail: jatristanchoo@udistrital.edu.co).

L. F. Vargas-. Profesor Titular del proyecto curricular de Ingeniería Industrial de la Universidad Distrital Francisco José de Caldas. Docente investigador del grupo de investigación DIMSI. (e-mail: lufvargast@ udistrital.edu.co).

L. E. Contreras-Bravo. Profesor Titular del proyecto curricular de Ingeniería Industrial de la Universidad Distrital Francisco José de Caldas. Director del grupo de investigación DIMSI. (e-mail: lecontrerasb@) udistrital.edu.co). representation of the world. During schooling, the development of this skill is integrated with the mathematics teaching curriculum, but mathematics generally focuses on the algorithmic process of solving non-spatial mathematical problems, whether in early childhood, formal education or at university. The engineering drawing is based on the ability to manipulate objects and ideas mentally (using spatial skills), to carry out the design process of any product or process.

In this paper the first part shows a bibliography revision of the state of the art. In the second part shows the results obtained in the application of different traditional techniques for the spatial skills improvement, identifying the favorable points, which are used as requirements to development of a new software. The final part presents the algorithm designed, the implementation and the impact on a test group is measured.

The results obtained show that this new computer tool allows the learning curve and the development of spatial skills to be significantly increased.

Index Terms - Computer aided drawing (CAD, Information and Communication Technologies (ICT), Pedagogy in engineering drawing, Spatial skills

\section{INTRODUCCIÓN}

QEGÚN [1] la habilidad espacial es la capacidad de percibir

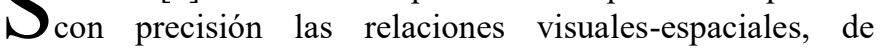
transformar estas percepciones, y de recrear algunos aspectos de la experiencia visual sin la presencia de los estímulos correspondientes.

En el desarrollo de la ingeniería la representación de ideas por medio de dibujos o esquemas ha sido fundamental. El lenguaje escrito o hablado no tiene la capacidad descriptiva que un dibujo puede tener [2]. El avance en la tecnología ha permitido que el proceso de diseño haya tenido una revolución en los últimos años, permitiendo la creación de ambientes virtuales tridimensionales y sistemas integrados de desarrollo [2]. La incursión de las computadoras ha mejorado de manera sustancial el proceso de diseño, pero las habilidades necesarias del ingeniero encargado a realizar un desarrollo son las mismas [1].

Los ingenieros siempre van a tener que generar, interpretar o verificar dibujos, esquemas y/o gráficas que en muchos 
casos corresponderán a elementos tridimensionales [2]. La diferencia de tiempo, metodología y profundidad para el desarrollo de habilidades espaciales en la primera infancia y en la escolarización primaria y secundaria, genera que se presenten muy disparejos niveles de desarrollo al momento de ingresar a la universidad [3].

También se han presentado cambios significativos en los pénsums de ingeniería, en la segunda parte del siglo $\mathrm{XX}$, el espacio disponible en cursos para desarrollar estas habilidades podía a llegar a ser del orden de 2 a 3 (p. ej. dibujo técnico o de ingeniería, geometría descriptiva, dibujo de máquinas, etc.). Los pénsums modernos de Ingeniería incluyen en la actualidad tan solo un curso, integrado con el aprendizaje del uso de alguna herramienta CAD [3]. Debido a esta necesidad existen numerosos estudios (p. ej. [1], [4] y [5]) de diferentes investigadores e instituciones que buscan la metodología que permita a los estudiantes nivelar y aumentar su habilidad espacial rápidamente.

Según lo planteado en [6], la enseñanza se puede dividir en dos tipos en pasiva y activa, refiriéndose al rol que el estudiante cumple durante su propia formación. Entre las metodologías de enseñanza pasiva se tiene: la recepción verbal (leer, oir) y recepción visual (mirar imágenes, ver pelicular, asistir a una exhibición, ver una demostración o ver un problema in situ). Mientras que la enseñanza activa tiene actividades como: participar en una discusión, dar una conferencia, hacer una presentación teatral, simular una experiencia real o construir algo. La enseñanza pasiva logra tener niveles de retención inferiores al 50\%, mientras que la enseñanza activa obtiene niveles superiores al 70\%. Estos tipos de resultados han sido demostrados por varios autores como [1], [7], [8] y [9].

Los resultados mostrados en [6] están basados en el modelo de estilo de aprendizaje de Kolb ([10] y [11]). Kolb propone que para aprender algo se debe trabajar o procesar la información recibida, partiendo de: una experiencia directa y concreta o bien de una experiencia abstracta (por ejemplo, de una lectura). Estas experiencias se convierten en conocimiento cuando se elaboran de manera reflexiva o experimentando de forma activa. El aprendizaje óptimo ocurre cuando se trabajan de manera consecutiva cada una de las fases: Actuar (Experiencia concreta), Reflexionar (observación reflexiva), Teorizar (Conceptualización abstracta) y Experimentar (Experimentación activa). La forma de conseguir que los estudiantes puedan llegar a traspasar las cuatro etapas descritas por Kolb durante su proceso educativo es el uso de laboratorios o prácticas de ingeniería [9].

En todo proceso de diseño tanto de productos como de técnicas o métodos didácticos, debe iniciar desde la identificación de las necesidades del usuario final. En el caso de la didáctica es necesario establecer cuál es la necesidad que se desea cubrir, tratando de incluir la mayor cantidad de información sobre el proceso formativo que se necesita y cuál es la aplicación de esa formación en la vida profesional del estudiante [7].

En [12] se realizó un estudio donde se aplicaron diferentes técnicas (tradicionales y no tradicionales) para evaluar el desarrollo de habilidades espaciales en estudiantes de primer semestre de ingeniería.

El estudio en [12] consistió en la medición de la condición inicial de desarrollo de habilidades espaciales por medio de la aplicación de la prueba estandarizada MRT (Mental Rotation Test), aplicación durante el semestre académico de manera independiente para cada grupo de estudio de 6 técnicas diferentes. Una vez concluido el semestre, se procedió a aplicar de nuevo la prueba MRT, donde se obtuvo como resultado de análisis principal el mostrado en la Fig. 1.

La Fig. 1 muestra que muestra claramente que las técnicas basadas en la interacción ojo mano, tienen la mayor facilidad y capacidad para el desarrollo de habilidades espaciales (solidos impresos 3D). Pero esto implica la necesidad de tener a disponibilidad material prediseñado disponible para los estudiantes, lo cual limita su aplicación en lugares diferentes al aula o laboratorio [12]. De la Fig. 1, también se puede extrapolar que el software especializado (SolidWORKS para el caso de [12]), no garantiza un desarrollo adecuado de habilidades espaciales, debido principalmente al nivel de complejidad de uso que una herramienta de esta categoría tiene [12].

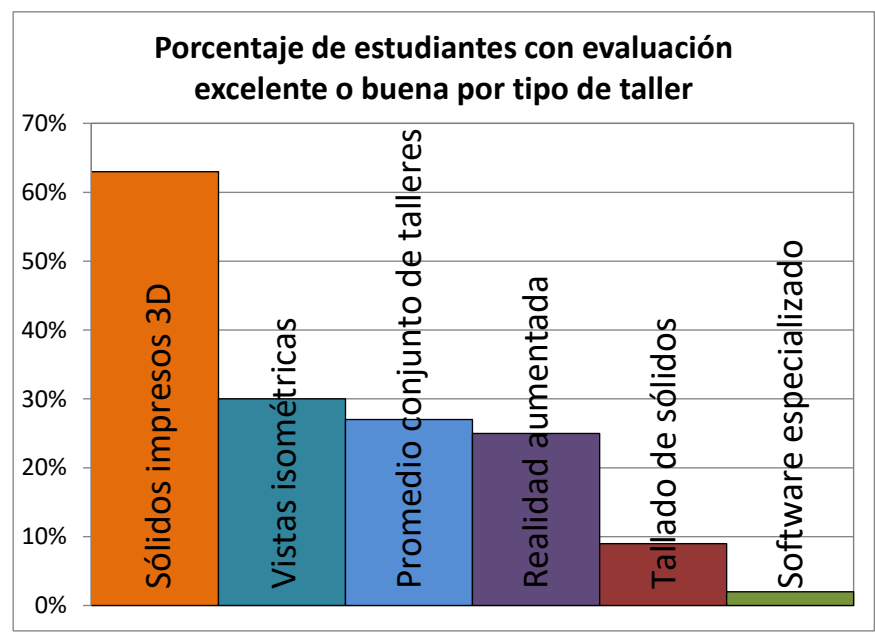

Fig. 1. Estudiantes con evaluación excelente o buena, categorizada por tipo de taller. Fuente: Adaptada de [12].

Una solución informática especializada en desarrollo de habilidades espaciales, con las características adecuadas permitiría que los estudiantes puedan trabajar en cualquier lugar, realizar manipulación virtual de objetos de muy variadas tipologías y niveles de complejidad, además de permitir la generación de nuevos objetos por parte de los usuarios.

En este artículo se describe el proceso de diseño de una herramienta de informática independiente CAD, orientada al desarrollo de habilidades espaciales, intuitivo, de uso libre y orientado al uso dentro y fuera del aula de clase. Este software integra los resultados de [12], que mostraron la necesidad de integración ojo mano y vistas isométricas.

\section{REVISIÓN DE TECNOLOgÍAS ACTUALES DisPoniBles}

Actualmente es posible encontrar muy diferentes softwares 
de uso libre o con pago de licencia, todos con variados niveles de complejidad y uso, para el desarrollo de habilidades espaciales o enseñanza del Dibujo en Ingeniería. Por ejemplo, [1] y [4], reportan alrededor de 30 aplicaciones y sitios WEB, diseñados para el desarrollo de habilidades espaciales.

Estos programas están diseñados para impactar en un grupo específico de estudiantes y con metodologías diferentes de uso. Pero se puede establecer que existen dos tipos de software específicos.

1. Aplicación basada en la solución de problemas preplanteados, mediante la variación de la visualización y la selección adecuada de la respuesta correcta (ver Fig. 1).

2. Software con dibujo limitado por parte de los usuarios con el fin crear algunas figuras, generalmente isométrico, por medio de vistas ortogonales o por medio de bloques individuales (ver Fig. 2)
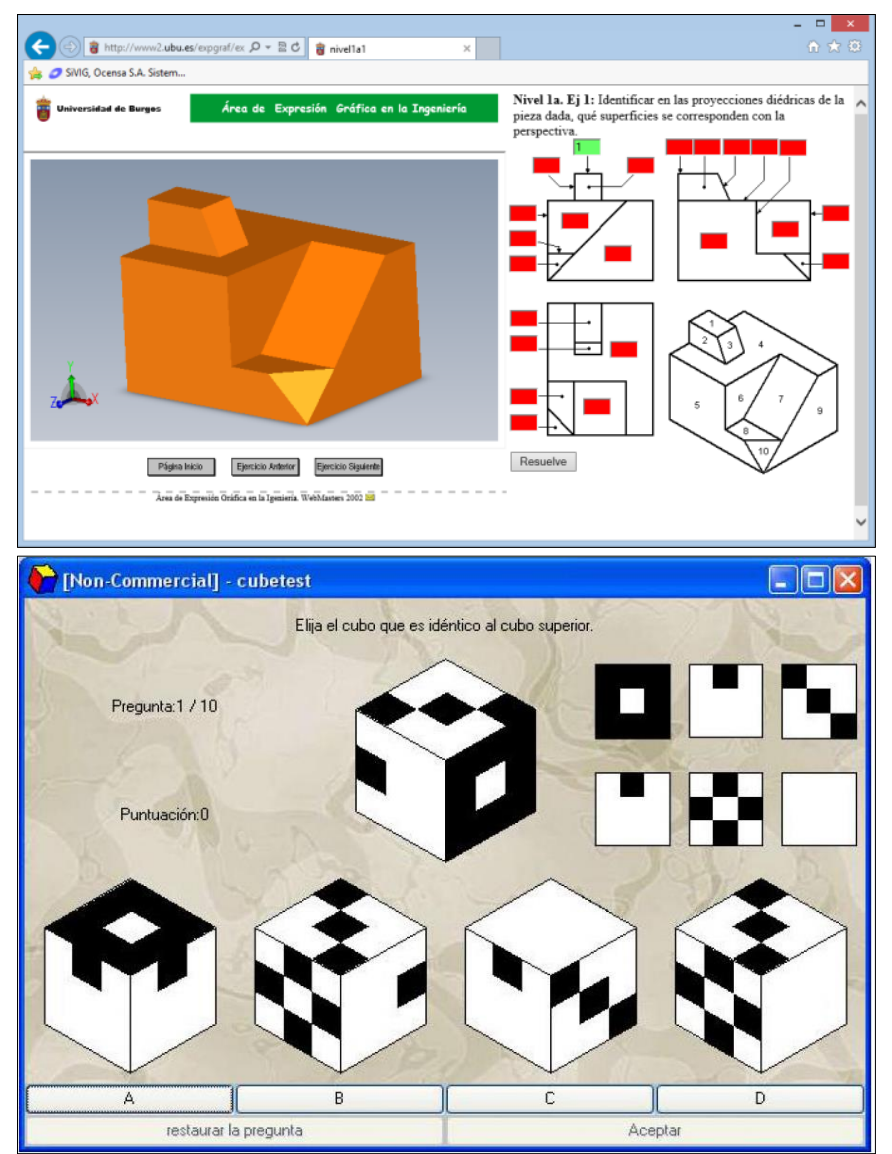

Fig. 2 Software de solución de problemas pre-planteados. Arriba: Aprendizaje Innovador de Visualización de Piezas mediante Taller Virtual. Abajo CubeTest (Joe van de Oever).

Los resultados obtenidos en [1], [4] y [5], muestran que el uso de software especializado ayuda en el desarrollo de habilidades espaciales. Los programas diseñados alrededor de problemas pre-planteados (estáticos) generan que los estudiantes entre rápidamente en monotonía y pierda el interés [4], además que la baja interactividad con los usuarios no permite que se desarrollen de manera adecuada las cuatro etapas del aprendizaje del modelo Knob [10].

Mientras el software tipo dibujo limitado permite una mayor interactividad de los usuarios y por ello un paso más
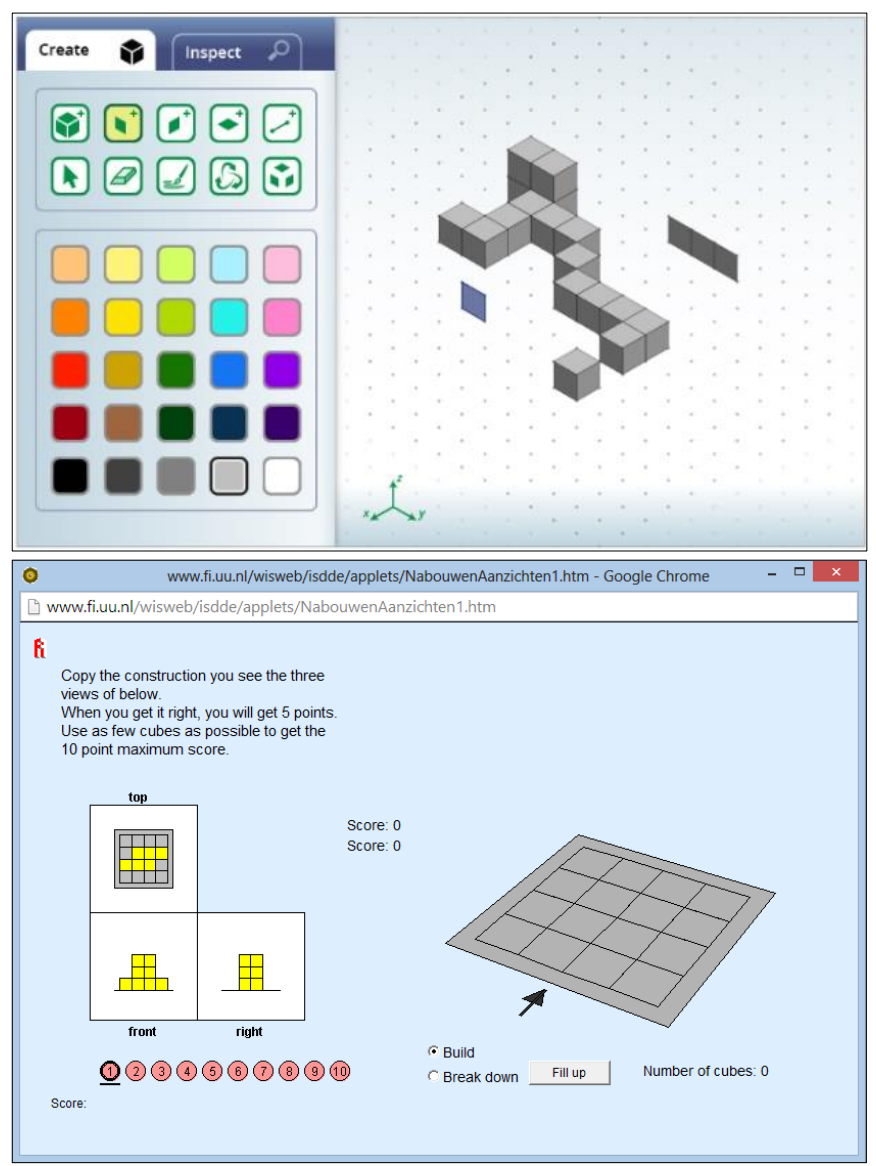

Fig. 3. Software de construcción de figuras Arriba. Isometric Drawing Tool (http://illuminations.nctm.org/), Abajo. Building with blocks (Freudenthal Institute for Science and Mathematics Education).

regular por las etapas de aprendizaje, pero al tener limitaciones de dibujo limita los posibles tipo y dificultad de ejercicios a desarrollar [5].

El algoritmo planteado en [13], tiene como desventaja la baja confiabilidad que presenta y la necesidad de conocer por completo las leyes de proyección isométrica para lograr obtener la representación tridimensional. Esto implica un entrenamiento significativo lo que conlleva a tener las limitaciones mostradas en [12] con el uso de software especializado (ver Fig. 3).

Partiendo del estudio realizado [13], buscando optimizar las características de aprendizaje de Knob, la técnica tradicional de desarrollo de habilidades espaciales por dibujo de objetos isométricos y con el objetivo de permitir una mayor integración con el estudiante sin tener la necesidad de incrementar significativamente las necesidades de conocimientos previos, se plantearon las siguientes características básicas del software a diseñar:

1. Dibujar objetos en proyección isométrica directamente, con un método de visualización e interpretación tridimensional [12]

2. Incrementar el nivel de interactividad respecto a las técnicas clásicas de dibujo en papel o en sistemas informáticos [5]

3. Aumentar el nivel didáctico del proceso de dibujo frente a los sistemas existentes usados en el aula [1] 
4. Permitir el uso de este nuevo software dentro y fuera del salón de clase por parte del estudiante $\mathrm{y} / \mathrm{o}$ profesor de manera expositiva [4]

Para el diseño del nuevo software se planteó la implementación en dos etapas. La primera etapa fue la selección de una herramienta de dibujo básico, con la cual el usuario pudiera realizar de manera simple un dibujo en proyección axonométrica de isometría. La segunda etapa se encargaría de realizar el cálculo inverso interactivo de obtención del objeto tridimensional equivalente y con representación en vistas ortogonales

\section{A. Sistema Base de Dibujo}

Como sistema base del software, se planteó el uso de una plataforma CAD (Sistema de diseño asistido por computador por sus siglas en inglés), para garantizar las condiciones adecuadas de calidad de dibujo. Se realizó un recorrido por diferentes softwares disponibles con licencia comercial y libre. Todas las herramientas CAD evaluadas no poseen la capacidad de generar una representación 3D desde el dibujo de una vista isométrica, por lo tanto, esto necesario programarlo e interconectarlo mediante el API (interfaz de programación de aplicaciones por sus siglas en inglés) de cada aplicación.

En la Tabla I se tiene una evaluación realizada por los autores de diferentes soluciones CAD disponibles. En la columna Uso, se tiene una evaluación de 1 a 5 con el tiempo de capacitación necesario (asociado con el nivel de dificultad de la herramienta, 4 es el que necesita un nivel superior de capacitación para su uso adecuado), la columna API determina si la aplicación tiene la posibilidad de ser programada o personalizada, la columna Isométrico determina si en el software es posible el dibujo en 2D con axonometría isométrica. La columna Características, determina si con la evaluación realizada es posible llegar a cumplir las características mínimas de la aplicación planteadas en el numeral anterior.

TABLA I

COMPARATIVA DE HERRAMIENTAS CAD DISPONIBLES PARA IMPLEMENTACIÓN DEL SISTEMA DE CÁLCULO INVERSO ISOMÉTRICO

\begin{tabular}{lcccccccc}
\hline \hline \multirow{2}{*}{ Software } & \multirow{2}{*}{ Libre } & \multirow{2}{*}{ Uso } & \multirow{2}{*}{ API } & Dibujo & \multicolumn{4}{c}{ Características } \\
& & & & Isométrico & 1 & 2 & 3 & 4 \\
\hline AutoCAD & - & 3 & $\mathrm{X}$ & $\mathrm{X}$ & $\mathrm{X}$ & - & $\mathrm{X}$ & - \\
SolidWorks & - & 4 & $\mathrm{X}$ & - & - & - & $\mathrm{X}$ & $\mathrm{X}$ \\
ZwCAD & - & 3 & $\mathrm{X}$ & $\mathrm{X}$ & $\mathrm{X}$ & - & $\mathrm{X}$ & - \\
FreeCAD & $\mathrm{X}$ & 4 & - & - & $\mathrm{X}$ & - & $\mathrm{X}$ & $\mathrm{X}$ \\
LibreCAD & $\mathrm{X}$ & 2 & - & $\mathrm{X}$ & $\mathrm{X}$ & - & $\mathrm{X}$ & $\mathrm{X}$ \\
SketchUp & $\mathrm{X}$ & 1 & $\mathrm{X}$ & - & - & - & - & $\mathrm{X}$ \\
BabaCad & $\mathrm{X}$ & 2 & $\mathrm{X}$ & $\mathrm{X}$ & $\mathrm{X}$ & - & $\mathrm{X}$ & $\mathrm{X}$ \\
\hline \hline
\end{tabular}

De los resultados obtenidos en la Tabla I se tiene que característica más difícil de implementar con CAD ya existente es el nivel de interactividad, pues los diferentes API no lo permiten de manera adecuada, pese a que la mayoría presenta implementación de eventos, no es posible tener el suficiente control sobre la interfaz del usuario.

Debido a que el uso de una herramienta ya preexistente no permitiría cumplir con las características mínimas planteados, se procedió a diseñar un sistema CAD independiente básico bidimensional con las herramientas mínimas necesarias para realizar un proceso de dibujo técnico, garantizando así las necesidades de capacitación al mínimo nivel y con un alto nivel de interactividad.

Esta herramienta $\mathrm{CAD}$ diseñada tiene implementados las siguientes funciones básicas: guardar y recuperar dibujos, dibujo de líneas por aproximación a construcciones a mano alzada, manejo de entidades vectoriales, copiar y pegar entidades, recortar y extender hasta la más cercana, uso de capas (que agrupan características como color, espesor y tipo de línea), cuadricula ortogonal, cuadricula isométrica y creación de relaciones geométricas básicas entre entidades (ortogonal, paralela, colineal y distancia). El software diseñado fue nombrado como SotDIN, acrónimo de SOftware de Tablero Digital para INgeniería (ver Fig. 4), partiendo de la idea del desarrollo de una plataforma base que puede evolucionar en otras áreas del conocimiento de ingeniería como: las matemáticas, física, mecánica de materiales, etc.

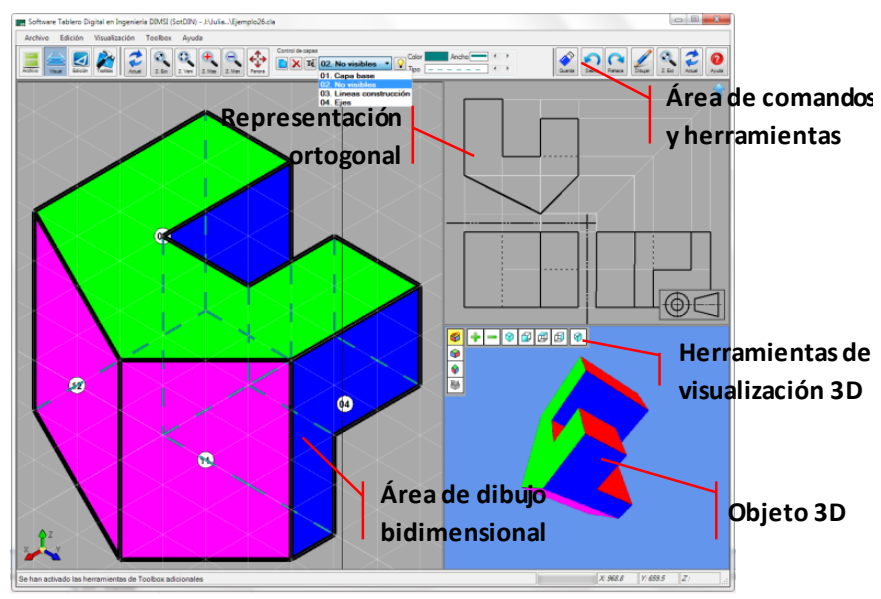

Fig. 4. Estructura básica de SotDIN

El desarrollo del sistema básico de dibujo en SotDIN, permite llegar a solucionar problemas típicos de representación básica en dibujo de ingeniería, sin tener el objetivo de ser una herramienta de dibujo de nivel profesional, pues su objetivo principal es dar la interfaz de usuario y gráfica para el proceso de representación 3D desde una vista isométrica y el usuario final son estudiantes de ingeniería (uso didáctico). Entre los tipos de dibujo que pueden ser realizados en SotDIN se tienen los mostrados en la Fig. 5: dibujo de representaciones isométricas (Fig. 5a y Fig. 5b), proyecciones oblicuas (cavalier y cantilever en la Fig. 5c y perspectiva con diferentes puntos de fuga en la Fig. 5d) y generación de representaciones multivista ortogonal (ortográficas), incluyendo vistas auxiliares como se muestra en la Fig. 5e y Fig. 5f 
a) Dibujo sin asistente de dibujo

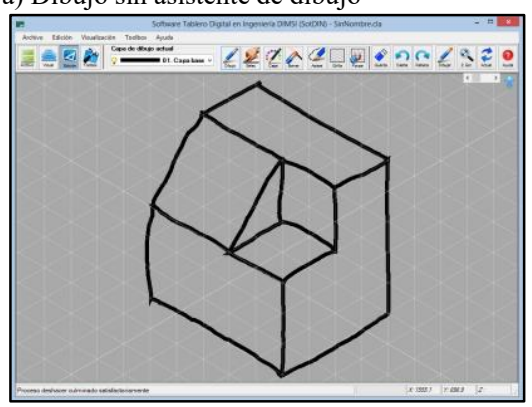

d) Dibujo en perspectiva

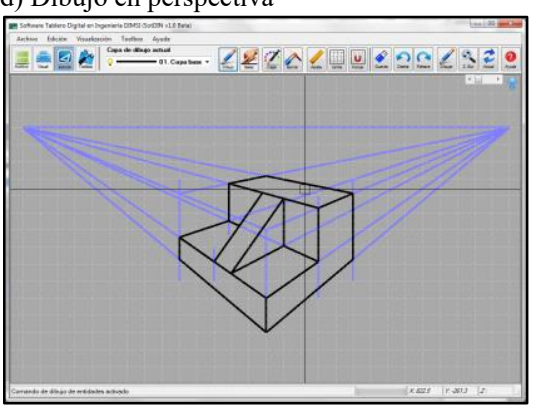

b) Dibujo con asistente de dibujo activado

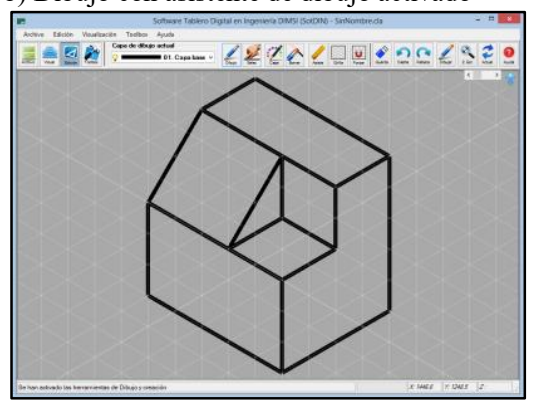

e) Generación de tercera vista

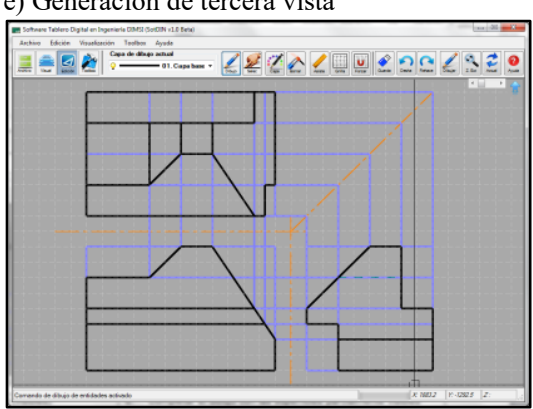

c) Vista oblicua tipo Caballera

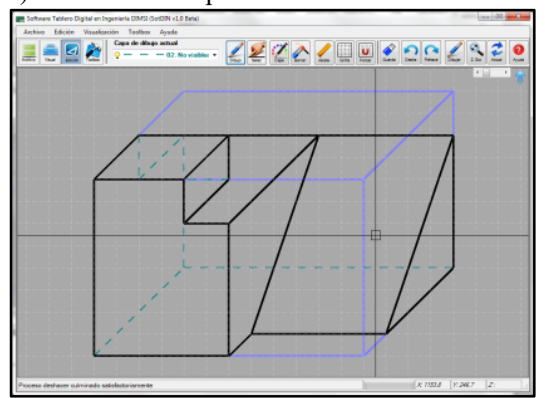

f) Generación de vista auxiliar

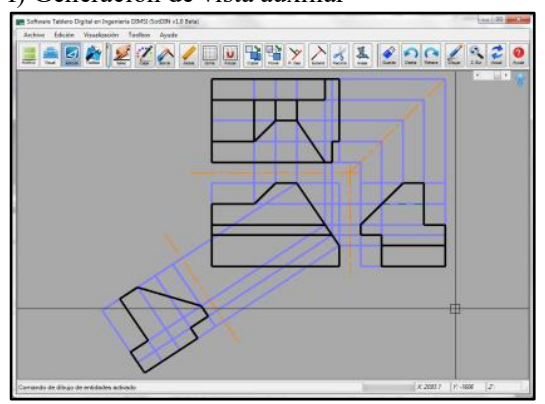

Fig. 5. Aplicaciones del software SotDIN en su módulo CAD bidimensional, para la solución de problemas de Dibujo en Ingeniería.

Con el objetivo de cumplir la característica de permitir la realización de sesiones expositivas por parte del profesor y/o estudiantes usando el software, se estableció como característica adicional la necesidad de poder ser usado en sistemas táctiles o de tablero digital. Un tablero digital consiste en un sistema de proyección de la pantalla del computador (video beam o televisor) con un método de apuntamiento y selección (marcador infrarrojo, ultrasónico o sistema táctil), cuya característica principal se puede resumir como sustitución del ratón. En la Fig. 6 se puede observar SotDIN trabajando integrado con el tablero digital. SotDIN tiene un diseño de la interfaz de usuario, está pensada para ser usada en clase para realizar explicaciones y ejercicios.

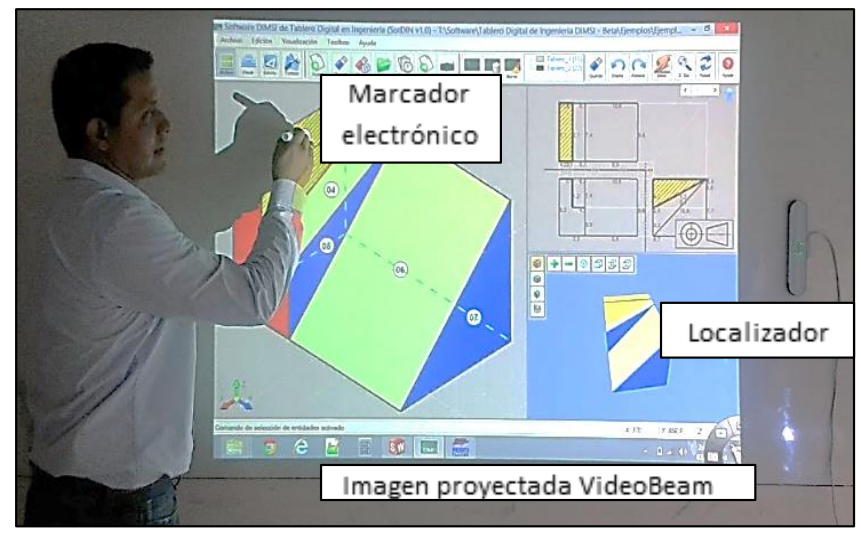

Fig. 6. Software SotDIN integrado con el tablero digital eBeam Edge

\section{B. Diseño Módulo de Interpretación Isométrica}

Con el sistema base de dibujo construido, se procedió a crear una biblioteca de vínculos dinámicos (.dll) que se acopla con la aplicación base. Esta librería permite a SofDIN identificar desde una proyección isométrica las diferentes superficies tridimensionales que componen el sólido representado.
El principal problema del dibujo en ingeniería es cómo representar objetos tridimensionales en un medio que es bidimensional: papel o la pantalla de un computador, garantizando que la representación sea lo más precisa, universal, clara e inequívoca posible. La visión humana deforma los objetos haciéndolos aparentar un tamaño mayor entre más cercanos al observador con respecto a los que se encuentran alejados, esto se conoce como perspectiva. La solución para evitar el efecto de la perspectiva, fue la de ubicar el observador teóricamente en el infinito, lo que origino las proyecciones axonométricas. En la proyección axonométrica se tiene que los rayos de proyección son paralelos entre sí y perpendiculares al plano de proyección [2].

La proyección en perspectiva asemeja perfectamente la forma "real" como el ojo humano percibe el mundo que lo rodea, pero tiene una serie de inconvenientes para los propósitos de la ingeniería como lo son: a) el dibujo en papel y computacionalmente son difíciles de obtener, b) las relaciones entre las dimensiones del objeto real y el representado se pierden y c) dependen completamente de la localización del observador con respecto al objeto [2]. Mientras que las proyecciones axonométricas no tienen la capacidad de la perspectiva para imitar la visualización humana, pero en cambio presenta las siguientes ventajas [2]:

- Axonometrías presentan una ventaja frente a la representación multivista, es que muestran más características del objeto (típicamente se pueden mostrar 3 vistas) en cada proyección, pero con la necesidad de deformar ángulos y longitudes.

- $\mathrm{Al}$ ser una proyección paralela las características que sean paralelas del objeto real, serán paralelas en la representación

- No hay puntos de fuga. Por lo tanto, el espectador verá la misma representación sin importar la distancia del punto desde donde se observe. 
- Los objetos distantes conservan su tamaño, independientemente de la ubicación del observador

- Existen reglas definidas para las proyecciones axonométricas. Por lo tanto, la generación de vistas axonométricas es computacionalmente muy ligeras

Matemáticamente para construir una proyección axonométrica, el objeto debe ser rotado en uno o dos de sus ejes principales para tener una inclinación con respecto al plano horizontal y/o vertical y luego proyectarlo sobre el plano de proyección. Si se supone que el plano de proyección es el plano $x y$, una vez girado el objeto con los ángulos establecidos para la proyección axonométrica, la coordenada $z$ es despreciada, obteniendo así la vista axonométrica bidimensional final.

Para obtener la rotación del solido alrededor del eje $y(\phi$ grados) y luego ( $\theta$ grados) alrededor del eje $x$, se debe hacer una doble transformación. En (1), se muestra la matriz de transformación $(T)$ necesaria para la doble rotación y proyección en el plano $x y$, la cual corresponde a una transformación homogénea del grupo de rotaciones en $R^{3}$ $\mathrm{SO}(3)$ [14].

$$
T=\left[\begin{array}{ccc}
\operatorname{Cos} \phi & 0 & -\operatorname{Sin} \phi \\
0 & 1 & 0 \\
\operatorname{Sin} \phi & 0 & \operatorname{Cos} \phi
\end{array}\right]\left[\begin{array}{ccc}
1 & 0 & 0 \\
0 & \operatorname{Cos} \theta & \operatorname{Sin} \theta \\
0 & -\operatorname{Sin} \theta & \operatorname{Cos} \theta
\end{array}\right]\left[\begin{array}{ccc}
1 & 0 & 0 \\
0 & 1 & 0 \\
0 & 0 & 0
\end{array}\right]
$$

Resolviendo la multiplicación matricial se obtiene:

$$
T=\left[\begin{array}{ccc}
\operatorname{Cos} \phi & \operatorname{Sin} \phi \cdot \operatorname{Sin} \theta & 0 \\
0 & \operatorname{Cos} \theta & 0 \\
\operatorname{Sin} \phi & -\operatorname{Cos} \phi \cdot \operatorname{Sin} \theta & 0
\end{array}\right]=\left(x_{p}, y_{p}, 0\right)
$$

El sistema (2) tiene que para las tres direcciones principales $(X, Y, Z)$, la proyección equivalente para cada par de puntos bidimensionales $\left(x_{p}\right.$ y $\left.y_{p}\right)$ es:

$$
\begin{aligned}
x_{p} & =X \cdot \operatorname{Cos} \phi+Y \cdot 0+Z \cdot \operatorname{Sin} \phi \\
& =X \cdot \operatorname{Cos} \phi+Z \cdot \operatorname{Sin} \phi \\
y_{p} & =X \cdot \operatorname{Sin} \phi \cdot \operatorname{Sin} \theta+Y \cdot \operatorname{Cos} \theta-Z \cdot \operatorname{Cos} \phi \cdot \operatorname{Sin} \theta
\end{aligned}
$$

Si se escogen los ángulos de rotación de $\phi=45^{\circ}$ alrededor del eje $y$, y un ángulo $\theta=35^{\circ}$ con respecto al eje $x$, se obtiene una proyección isométrica, que genera un ángulo entre los ejes principales de $120^{\circ}$ y una deformación uniforme de las tres direcciones. En (3) se puede ver uno de los problemas fundamentales de la representación isométrica, un punto en el espacio no tiene una única representación en el plano de proyección, esto origina el problema de ambigüedad, donde puntos que pertenecen a superficies diferentes (posición $X, Y, Z$ diferentes) aparecen ubicados en las mismas coordenadas de la proyección $\left(x_{p}, y_{p}\right)$, como se puede ver en Fig. 7.

Para encontrar desde la proyección isométrica, el objeto tridimensional original de cada punto es necesario solucionar
(3) o Ecuación de Cálculo Inverso Isométrico (ECII). Esto origina un sistema de dos ecuaciones y tres variables independientes, el cual no tiene solución directa. Como método de solución entonces se propone el desglose del solido

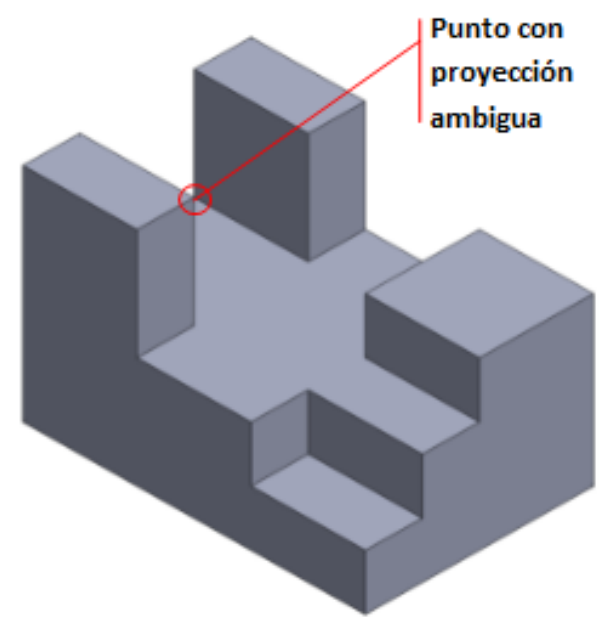

Fig. 7. Ejemplo de punto con proyección ambigua en una representación isométrica

en superficies independientes, usando como condición geométrica característica de ser una superficie plana, además de una condición de inclinación con respecto al sistema coordenado.

En la Tabla II se establece en la columna Condición, como se limita los posibles valores de una variable, permitiendo la solución de la ECII, como se muestra en la columna Sistema solución.

De la Tabla II se obtiene que los planos que sean paralelos a los principales (planta, frontal y lateral) quedan definidos completamente, pero basadas en un sistema coordenado indefinido, originado por el sistema de proyección. Mientras los planos secundarios (inclinados u oblicuos), solo se pueden definir basados en las condiciones de tres puntos conocidos del plano en estudio, generalmente originados por algún(os) plano(s) principal(es) colindante(s).

\section{Algoritmo de Ajuste de Superficies y Cierre de Solido}

Antes de iniciar el proceso de cierre de solido es necesario ejecutar un algoritmo de ajuste que permite asegurar que las relaciones geométricas básicas de los planos que forman el sólido se conserven. Este algoritmo verifica relaciones de paralelismo, perpendicularidad, colinealidad e igualdad en los bordes que definen el plano.

La técnica de cálculo inverso de planos solo permite solucionar la ubicación de planos fuera de un origen común. La siguiente tarea es unir de manera adecuada las diferentes superficies para obtener la representación del sólido buscada. Para ello el algoritmo verifica todos los vértices existentes en la representación isométrica, vinculando cada una de las superficies que intervienen, es importante anotar que el algoritmo identifica los posibles puntos ambiguos y restringe su uso para la reubicación, con el fin de eliminar los posibles errores de unión. 
TABLA II

COMBINACIÓN DE TIPOS DE INCLINACIÓN DISPONIBLES PARA PLANOS EN SÓLIDOS Y ECUACIONES DE CÁLCULO INVERSO

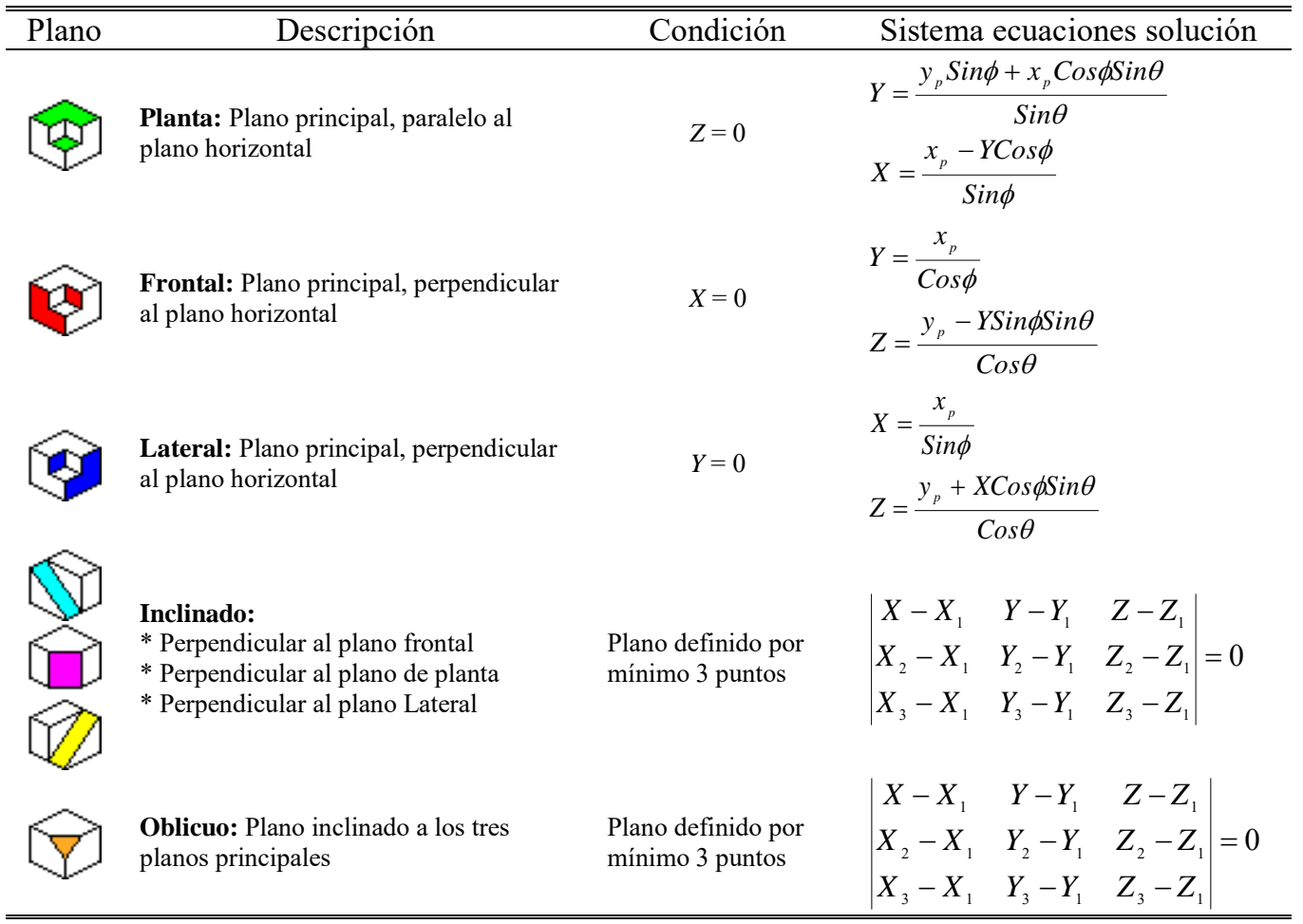

El proceso de cierre inicia al definir un plano principal inicial, el cual concreta el origen base para los demás planos. Con la información de vértices se procede a reubicar cada uno de los planos restantes, mediante la ubicación relativa de cada uno. El algoritmo de ajuste realiza varias alternativas de manera secuencial tratando de encontrar la mejor solución posible, para ello el sistema calcula un índice basado en el mínimo volumen posible y cambios de dirección sobre las definiciones de los planos. La solución que posea el mínimo indicador será tomada como solución del problema. En la Fig. 7 se muestra el proceso completo de creación de un sólido por cálculo inverso desde vista isométrica usando el software SotDIN.

\section{Características Software SotDIN}

SotDIN permite generar a partir de una representación isométrica, la representación tridimensional de planos interconectados, con la capacidad de movimiento mediante el apuntador (visualización tridimensional con rotación del objeto controlada por el usuario mediante el apuntador del sistema), tres vistas principales es sistema de proyección de tercer cuadrante con líneas ocultas, representación en proyección dimétrica y trimétrica estática (sin posibilidad de movimiento o cambio en los ángulos de proyección).

La generación del objeto tridimensional a partir de la proyección isométrica, se realiza paso a paso, por lo cual el usuario puede ver como cada uno de las superficies que se crean, se van integrando para formar el sólido final, tanto en la representación en vistas ortogonales, como en la vista tridimensional. En la representación del objeto en proyección isométrica se puede incluir los planos ocultos, mediante la creación de los mismos con líneas ocultas. Si no se incluyen los planos simplemente el sistema mostrara el objeto tridimensional sin esos planos. Esto es parte también de la estructura didáctica, pues ofrece un tipo de entrenamiento adecuado sobre el comportamiento de los planos no visibles.

Todos los objetos pertenecientes al solido (bordes y planos), están vinculados en la representación por vistas, isométrica y sólida, lo que genera que si el usuario seleccionada un objeto en cualquiera de estas vistas, se resaltara su representación equivalente en las otras dos. SotDIN posee además un método de exportación 3D basado en la estructura de intercambio de archivos dxf (Drawing Exchange Format), lo que permite que los dibujos sean importados por programas de dibujo profesional como AutoCAD y SolidWORKS para proseguir con los procesos de edición si así lo desea.

SotDIN fue desarrollado en plataforma Visual Basic .NET 2010, usando como sistema de dibujo la librería integrada de Microsoft GDI+ y como motor de visualización 3D el Framework Microsoft XNA 4.0. Los requerimientos mínimos son: Computador PC o compatible Pentium IV o superior, Sistema operativo Windows XP o superior, espacio en disco mínimo de $250 \mathrm{Mb}$ y memoria RAM de $1024 \mathrm{Mb}$. Estas características permiten que la aplicación sea de uso libre, con muy bajos requerimientos del sistema, por ejemplo, al ser compatible con sistemas operativos Windows, garantiza que puedan ser ejecutados en cerca del $88 \%$ de los computadores personales a nivel mundial [15]. 
a) Dibujar la representación isométrica

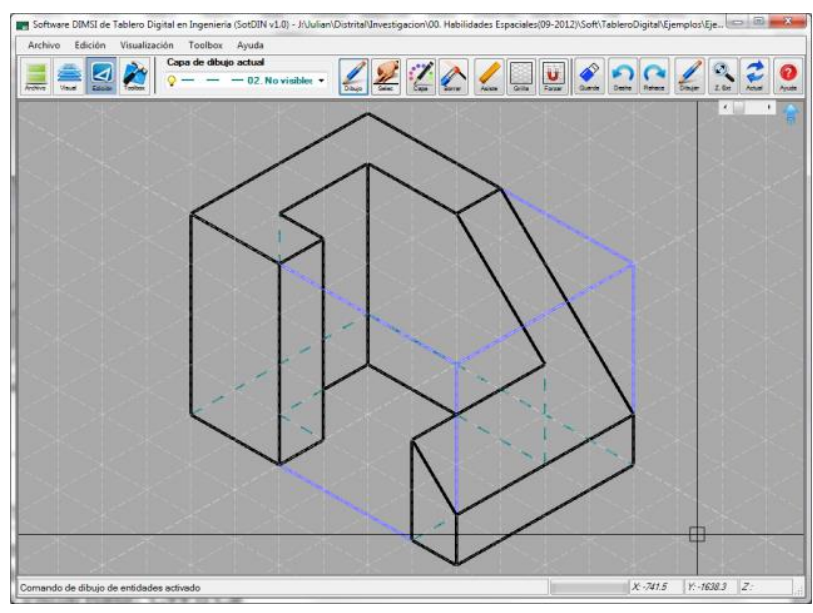

c) Definir las superficies y característica tipo (principales no visibles)

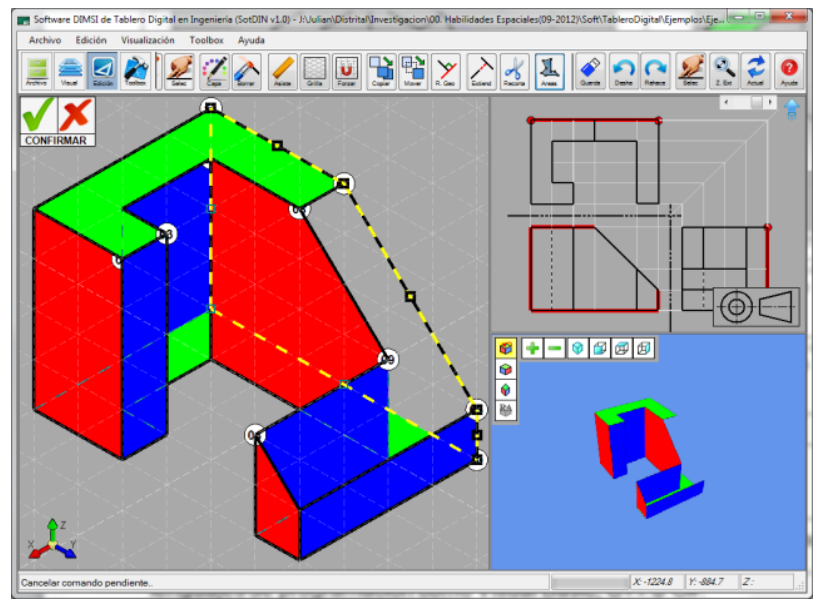

Fig. 8. Proceso de creación de solido por cálculo inverso desde el isométrico.

\section{IMPLEMENTACIÓN Y EVALUACIÓN DE SOTDIN}

Para evaluar el rendimiento y capacidad de SotDIN en fortalecer el desarrollo de habilidades espaciales se usaron dos instrumentos: encuesta de satisfacción y aplicación de pruebas estandarizadas de evaluación en el desarrollo de habilidades espaciales.

El universo usado para la evaluación de satisfacción y de desarrollo de habilidades espaciales correspondió a 40 estudiantes, de primer semestre de un total de 120 inscritos a Ingeniería de la Universidad Francisco José de Caldas

\section{A. Encuesta de Satisfacción del Usuario}

En la encuesta se realizaron 9 preguntas relacionadas directamente con el software, como se puede ver en la Tabla III. Estas preguntas van desde el uso en el salón de clase, pasando por la interfaz de usuario hasta resultados en el proceso formativo. En cada pregunta el estudiante podía proceder a calificar de 1 a 5 , donde 1 es muy insatisfecho y 5 es muy satisfecho. La encuesta se realizó mediante una aplicación de internet y de manera anónima por parte de los estudiantes. El promedio total en la encuesta arrojó un 4.1/5.0. b) Definir las superficies y característica tipo (principales)

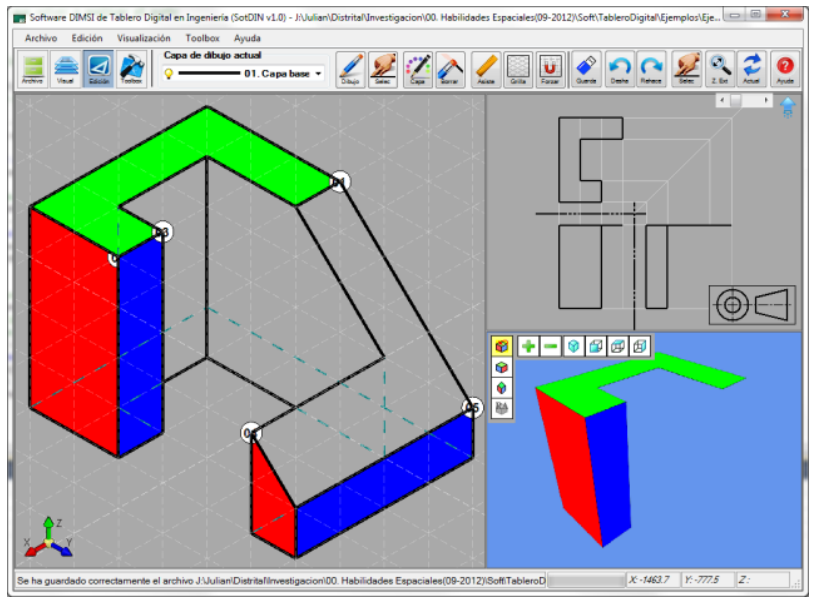

d) Definir las superficies y característica tipo (no principales)

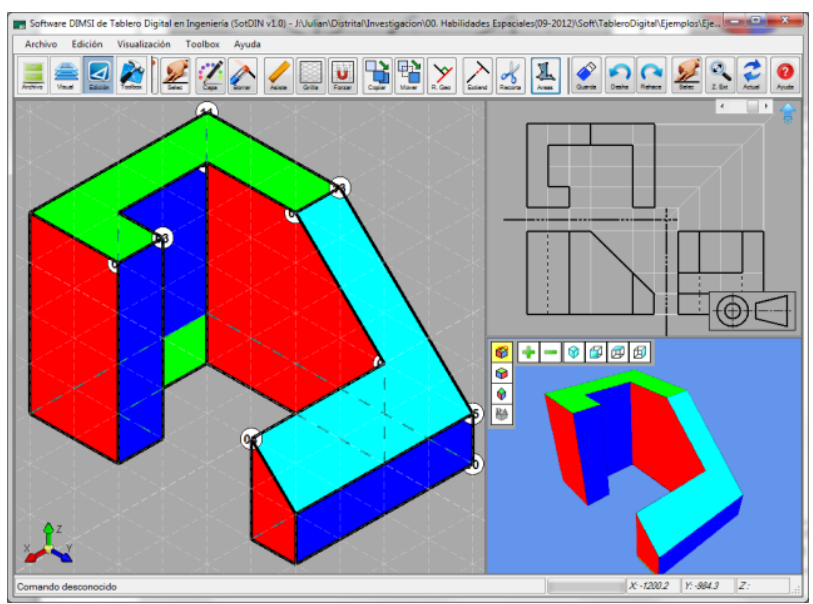

TABLA III

RESPUESTAS ASOCIADAS A SOTDIN CON RESPECTO A LA SATISFACCIÓN DE USO

\begin{tabular}{|c|c|}
\hline PREGUNTA & $\overline{\bar{X}}$ \\
\hline $\begin{array}{l}\text { 1. ¿Cómo considera el uso de esta aplicación en el salón de clase } \\
\text { para la solución de ejercicios tipo de Dibujo en Ingeniería? }\end{array}$ & 4.4 \\
\hline $\begin{array}{l}\text { 2. ¿Cómo considera que el uso de este software en clase, } \\
\text { permite entender mejor el procedimiento de solución de } \\
\text { problemas? }\end{array}$ & 4.3 \\
\hline 3. ¿Cómo es la calidad de la interfaz gráfica del software? & 3.4 \\
\hline 4. Con respecto a la integración entre el Tablero Digital y el & \\
\hline $\begin{array}{l}\text { Software DIMSI, ¿Cree usted que es buena para agilizar la labor } \\
\text { en clase y dar mayor claridad a los temas tratados en ella? }\end{array}$ & 4.5 \\
\hline $\begin{array}{l}\text { 5. ¿Usted recomendaría el uso de este software para el } \\
\text { desarrollo de talleres y temas de estudio por parte de sus } \\
\text { compañeros de próximos semestres? }\end{array}$ & 4.2 \\
\hline $\begin{array}{l}\text { 6. En el trabajo individual desarrollado por usted, ¿Este software } \\
\text { le ayudo a resolver dudas y clarificar conceptos? }\end{array}$ & 4.0 \\
\hline $\begin{array}{l}\text { 7. ¿Las imágenes o iconos usados en los botones de la barra de } \\
\text { herramientas son claros? }\end{array}$ & 4.0 \\
\hline $\begin{array}{l}\text { 8. ¿El procedimiento de dibujo es claro y tiene lógica, es fácil de } \\
\text { entender y aplicar para nuevos problemas? }\end{array}$ & 3.9 \\
\hline $\begin{array}{l}\text { 9. ¿Los resultados obtenidos luego de realizar un proceso de } \\
\text { dibujo, son útiles e importantes? }\end{array}$ & 4.1 \\
\hline PROMEDIO & 4.1 \\
\hline
\end{tabular}

\section{B. Resultados de Evaluación Habilidades Espaciales}

Para hacer medir como la implementación de esta herramienta y metodología en el curso de Dibujo en 
Ingeniería, ayudaron en el desarrollo de las habilidades espaciales por parte de los estudiantes se aplicaron dos test estandarizados, uno al inicio del curso y el otro al finalizar el curso.

El test inicial aplicado fue la prueba de rotación mental (MRT por sus siglas en inglés). La MRT es una de las pruebas con mayor nivel de aceptación y aplicabilidad a nivel mundial para la evaluación de habilidades espaciales, la cual fue desarrollada por [16]. Está diseñada para medir la actitud de las personas a reconocer los diseños de un objeto formado por el ensamble de objetos sencillos diferentes. La única diferencia entre el cuerpo original y el objeto a encontrar es una modificación del ángulo en el cual es visto [5].

En el test final y para garantizar que no se obtendrían resultados basados en el aprendizaje a la solución de problemas tipo de la MRT, se aplicó un test evaluación de habilidades espaciales diseñado para la valoración inicial de pilotos de combate del ejército estadounidense [17], la cual corresponde a la visualización en dos o tres vistas ortogonales y un magazín de posibles sólidos (con leves cambios entre sí) en representación isométrica girados en el espacio, donde solo uno corresponde al solido originario de las vistas presentadas.

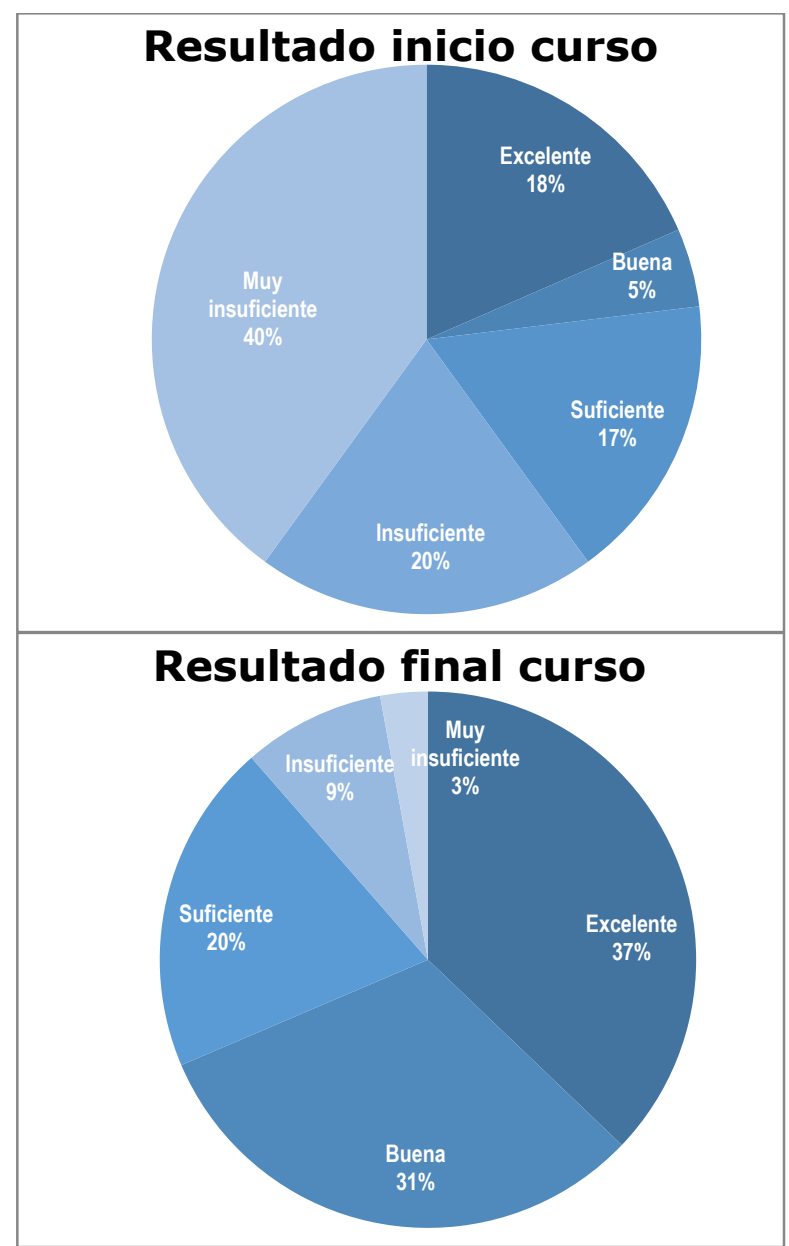

Fig. 9. Comparación de resultados pruebas de habilidades espaciales. Arriba. Resultados pre cursos y Abajo. Resultados al final del curso

La Fig. 9 muestra la comparación de resultados obtenidos en la prueba inicial y final. Se puede ver como los estudiantes antes de ingresar al curso de Dibujo en Ingeniería se tiene solo una calificación de Excelente y Buena del orden de 23\%, mientras que Muy Insuficiente e Insuficiente del orden de $60 \%$. En cuanto al rendimiento de los estudiantes una vez termino el curso y usado SofDIN como parte de la metodología del mismo, se tiene que Excelente y Buena suma $68 \%$, en tanto que Insuficiente y Muy Insuficiente es $12 \%$

\section{CONCLUSIONES}

De los resultados obtenidos en el grupo de análisis de estudiantes de primer semestre en Ingeniería de la Universidad Distrital Francisco José de Caldas muestran que el diseño e implementación de software especializado en el desarrollo de habilidades espaciales, permiten obtener una mejor curva de aprendizaje en cuanto a su velocidad y de mejor calidad. Según las evaluaciones de habilidades espaciales se triplica el porcentaje de personas con nivel superior a Bueno y se reduce cerca de 10 veces los estudiantes con nivel Muy insuficiente.

El tablero digital interactivo es una herramienta de hardware que permite que los desarrollos de teoría en clase sean más dinámicos y con menores tiempos de manejo operativo que de uso real didáctico. Uno de los inconvenientes que se presentan al implementar técnicas como el tablero digital, es la carencia de software diseñado para esta herramienta que se encuentre orientado a estudiantes de ingeniería. Teniendo en cuenta este aspecto se desarrolló una herramienta completamente independiente (SotDIN) y pensada desde un principio para ser acoplada a la tecnología de tablero digital, diseñada para el desarrollo de habilidades espaciales y uso dentro del aula de clase del curso de Dibujo en Ingeniería.

El diseño de SotDIN permite que se use la estructura base (sistema de dibujo y herramientas CAD bidimensional), sumada a una librería tipo toolbox, en diversos campos de la formación de ingenieros. En este caso se desarrolló un método de generación de solido 3D desde una representación isométrica, para el desarrollo de habilidades espaciales, pero sería posible diseñar nuevas toolbox, basadas en la estructura base y comunicación COM o NET, con las siguientes posibles aplicaciones:

- Matemáticas: Cálculo, algebra lineal, mediante la implementación de un sistema de reconocimiento caligráfico, cálculo de funciones, métodos numéricos de derivación e integración o métodos de manejo simbólico

- Física: Mecánica y de fluidos, modelamiento matemático de procesos físicos basados en la solución de las ecuaciones diferenciales numéricamente acoplados a los objetos gráficos.

- Análisis simple estructural: cerchas y estructuras simples en $2 \mathrm{D}$ y $3 \mathrm{D}$, con el dibujo de estructuras, aplicación de cargas y restricciones, para la solución mediante sistemas matriciales.

\section{REFERENCIAS}

[1] J. Martín Gutiérrez, "Estudio y evaluación de contenidos didácticos en el desarrollo de las habilidades espaciales en el ámbito de la ingeniería," Universitat Politècnica de València, Valencia (Spain), 2010. DOI: $10.4995 /$ Thesis/10251/7527

[2] G. Bertoline, E. Wiebe, N. Hartman, and R. William, Technical Graphics Communications. McGraw-Hill, 2009. 
[3] E. D. Jaimes and A. R. Vázquez, "Integrando el uso de habilidades espaciales y geométricas para el aprendizaje significativo del concepto de volumen de sólidos con estudiantes de dibujo técnico," Rev. CIENTÍFICA, pp. 462-466, 2013.

[4] C. Melgosa Pedrosa, "Diseño y eficacia de un gestor web interactivo de aprendizaje en ingeniería gráfica para el desarrollo de la capacidad de visión espacial," Universidad de Burgos, 2012.

[5] J. L. Saorín, R. Navarro, N. Martín, and M. Contero, "Expresión Gráfica en Arquitectura e Ingeniería y el desarrollo de visión y habilidades espaciales de los alumnos en las carreras técnicas," in Apega 2006: Asociación de profesores de Expresión Gráfica aplicada a la edificación, 2006.

[6] R. Chrobak, "The Globalization and the Engineering Teaching for the XXI Century," in Primer Congreso Argentino de Enseñanza en la Ingeniería, 1996.

[7] S. Caro and J. C. Reyes, "Prácticas docentes que promueven el aprendizaje activo en ingeniería civil," Rev. Ing., vol. 18, pp. 48-55, 2003.

[8] D. Ruiz Valencia, J. Magallón Gudiño, and E. Muñoz Díaz, "Herramienta de aprendizaje activo en las asignaturas de ingeniería estructural", IyU, vol. 10, no. 1, Oct. 2010.

[9] G. Fernández-Sánchez and M. Á. Millán, "Structural Analysis Education: Learning by Hands-On Projects and Calculating Structures," J. Prof. Issues Eng. Educ. Pract., vol. 139, no. 3, pp. 244-247, Jul. 2013. DOI: 10.1061/(ASCE)EI.1943-5541.0000155

[10] D. Kolb, and R. Fry. "Toward an Applied Theory of Experiential Learning." Theories of Group Process, 1975.

[11] J. E. Sharp, J. N. Harb, and R. E. Terry, "Combining Kolb Learning Styles and Writing to Learn in Engineering Classes," J. Eng. Educ., vol. 86, no. 2, pp. 93-101, Apr. 1997. DOI: $10.1002 /$ j.21689830.1997.tb00271.x.

[12] J. A. Tristancho, L. E. Contreras, and L. F. Vargas, "Evaluación de técnicas tradicionales y TIC para el desarrollo de habilidades espaciales en estudiantes de primer semestre de ingeniería industrial," Rev. Virtual Católica del Norte, vol. 43, pp. 34-50, 2014.

[13] P. Company, M. Contero, J. Conesa, and A. Piquer, "An optimisation-based reconstruction engine for 3D modelling by sketching," Comput. Graph., vol. 28, no. 6, pp. 955-979, Dec. 2004. DOI: 10.1016/j.cag.2004.08.007

[14] D. Salomon, The Computer Graphics Manual, 1st ed. London: Springer-Verlag London, 2011.

[15] Statista, "Cuota de mercado de los principales sistemas operativos a nivel mundial en el primer semestre de 2018," 2018. [Online]. Available: https://es.statista.com/estadisticas/576870/cuota-demercado-mundial-de-los-sistemas-operativos/.

[16] S. G. Vandenberg, and A. R. Kuse. "Mental Rotation, a Group Test of Three-Dimensional Spatial Visualization." Perceptual and Motor Skills 47, pp. 599-604., 1978

[17] Mechanical and Spatial Aptitude. Learningexpress, pp: 99-102, 118124, 127-130, 2001.

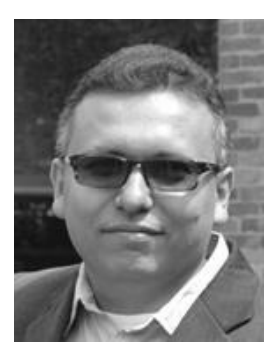

Julian Alfonso Tristancho Ortiz Ingeniero mecánico de la Universidad Nacional de Colombia (2000), con Maestría en Ingeniería Electrónica y de Computadores de la Universidad de Los Andes (2006) y Doctorado en Ingeniería de la Universidad de Los Andes (2012).

Él es profesor asistente de la Universidad Distrital "Francisco José de Caldas" desde 2011, adscrito al proyecto curricular de Ingeniería Industrial. Ha desarrollado su vida laboral alrededor de la docencia y del diseño de software especializado para ingeniería en variadas áreas y diferentes plataformas.

Las áreas de investigación principales del Dr. Tristancho donde tiene diversas publicaciones en revistas especializadas son, software y libros académicos: Diseño de software especializado de ingeniería, Modelamiento Numérico y
Simulación Física, Procesos de control y Automatización Industrial.

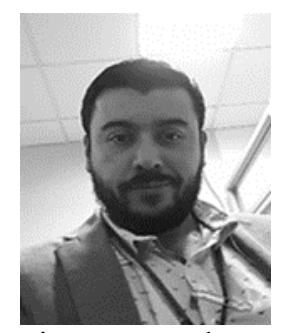

Luis F. Vargas nació en Sogamoso Boyacá, en 1977. Estudio ingeniería mecánica en la Universidad Nacional de Colombia sede Bogotá, graduado en el año 2001. Luego, en 2006 recibió el título de Magister en ingeniería mecánica - materiales y procesos de manufactura de la misma universidad. Acumula quince años de trabajo docente en varias universidades de Bogotá, trabajando en la formación de ingenieros industriales y mecánicos a nivel de pregrado y en especializaciones relacionadas con la automatización y la seguridad industrial; actualmente es docente de planta, categoría titular de la Universidad Distrital Francisco José de Caldas en Bogotá.

Los campos de interés están por la simulación y modelamiento computacional de sistemas, equipos y plantas. La ingeniería inversa y los sistemas de digitalización automática y desde luego, la docencia universitaria. Enmarcado en estos temas su producción abarca más de 25 artículos publicados en revistas indexadas, 9 libros de investigación y de uso docente $\mathrm{y}$ también algunas piezas de software para fortalecer el uso en las aulas de clase.

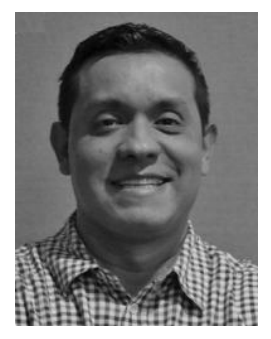

Leonardo E. Contreras Bravo. Nacido en Sincelejo (Sucre -Colombia) en 1977. Graduado como Ingeniero Mecánico de la Universidad Francisco de Paula Santander (Cúcuta) en 1999. Magister en Ingeniería Mecánica de la Universidad Nacional de Colombia (2005). Actualmente estudia el Doctorado en ingeniería en la Universidad Distrital Francisco José de Caldas.

Desde el año 2006, ha estado trabajando en la Universidad Distrital Francisco José de Caldas. Ha sido Coordinador de ingeniería Industrial y Director del grupo de investigación DIMSI (Diseño, modelamiento y simulación). Es autor de tres libros relacionados con el área de materiales y procesos de manufactura, así como de tres libros relacionados con el área de las TIC y la educación a nivel universitario. Autor de diferentes artículos del área de la ingeniería mecánica, tecnologías de la educación y ciencia de datos.

El profesor Contreras como investigador Junior ha trabajado en áreas como: Biomecánica, desarrollo de productos $\mathrm{y}$ procesos, nuevas tecnologías en educación, docencia universitaria y analítica de datos. Sus trabajos han sido presentados en eventos científicos y han sido publicados en revistas nacionales e internacionales. 